\title{
Thalamic Projection Fiber Integrity in de novo Parkinson Disease
}

\author{
P.J. Planetta, E.T. Schulze, E.K. Geary, D.M. Corcos, J.G. Goldman, D.M. Little, and D.E. Vaillancourt
}

\section{ABSTRACT}

BACKGROUND AND PURPOSE: Postmortem studies of advanced PD have revealed disease-related pathology in the thalamus with an apparent predilection for specific thalamic nuclei. In the present study, we used DTI to investigate in vivo the microstructural integrity of 6 thalamic regions in de novo patients with PD relative to healthy controls.

MATERIALS AND METHODS: Forty subjects (20 with early stage untreated PD and 20 age- and sex-matched controls) were studied with a high-resolution DTI protocol at 3T to investigate the integrity of thalamic nuclei projection fibers. Two blinded, independent raters drew ROIs in the following 6 thalamic regions: AN, VA, VL, DM, VPL/VPM, and PU. FA values were then calculated from the projection fibers in each region.

RESULTS: FA values were reduced significantly in the fibers projecting from the AN, VA, and DM, but not the VPL/VPM and PU, in the PD group compared with the control group. In addition, there was a reduction in FA values that approached significance in the VL of patients with PD. These findings were consistent across both raters.

CONCLUSIONS: The present study provides preliminary in vivo evidence of thalamic projection fiber degeneration in de novo PD and sheds light on the extent of disrupted thalamic circuitry as a result of the disease itself.

ABBREVIATIONS: $\mathrm{AN}=$ anterior nucleus; $\mathrm{DM}=$ dorsomedial nucleus; $\mathrm{FA}=$ fractional anisotropy; $\mathrm{PD}=$ Parkinson disease; $\mathrm{PU}=$ pulvinar; $\mathrm{SN}=$ substantia nigra; $\mathrm{VA}=$ ventral anterior nucleus; $\mathrm{VL}=$ ventral lateral nucleus; $\mathrm{VPL}=$ ventral posterior lateral nucleus; $\mathrm{VPM}=$ ventral posterior medial nucleus

$P$ $\mathrm{D}$ is a progressive neurodegenerative disorder with a slow, predictable course through vulnerable regions of subcortical and cortical gray matter. ${ }^{1}$ The pathologic hallmark of PD is the selective and substantial loss of dopaminergic neurons in the $\mathrm{SN}$ pars compacta. ${ }^{1,2}$ It is generally accepted that a reduction of do-

Received September 22, 2011; accepted after revision March 30, 2012. From the Departments of Applied Physiology and Kinesiology (P.J.P., D.E.V.), Neurology (D.E.V.), and Biomedical Engineering (D.E.V.), University of Florida, Gainesville, Florida; Departments of Neurology and Rehabilitation (E.T.S., E.K.G., D.M.C.), Kinesiology and Nutrition (D.M.C.), Physical Therapy (D.M.C.), and Bioengineering (D.M.C.), University of Illinois at Chicago, Chicago, Illinois; Department of Neurological Sciences (D.M.C., J.G.G.), Rush University Medical Center, Chicago, Illinois; Department of Veterans Affairs, VISN 17 Center of Excellence for Research on Returning War Veterans (D.M.L.), Waco, Texas; and Department of Psychiatry and Behavioral Science (D.M.L.), Texas A\&M Health Sciences Center, Bryan, Texas.

P.J.P. and E.T.S. contributed equally to this work.

This work was supported in part by grants from the Department of Defense/Congressionally Directed Medical Research Program (PT 075675) to D.M.L., Michael J. Fox Foundation (D.E.V.), and National Institutes of Health (R01-NS-52318 and R01NS-58487 to D.E.V.).

Please address correspondence to David E. Vaillancourt, PhD, Department of Applied Physiology and Kinesiology, University of Florida, PO Box 118205, Gainesville, FL 32611; e-mail: vcourt@ufl.edu

-- Indicates open access to non-subscribers at www.ajnr.org

http://dx.doi.org/10.3174/ajnr.A3178 pamine in the SN pars compacta underlies dysfunction within the direct basal ganglia pathway, ${ }^{3}$ which results in reduced excitation of cortical neurons by the thalamus. ${ }^{4}$ This increased inhibition of cortical-subcortical circuits in PD results in bradykinesia and various degrees of cognitive impairment. ${ }^{5-7}$ Thus, while dysfunction of subcortical structures such as the basal ganglia and thalamus have historically been conceptualized as a consequence of SN pars compacta degeneration, there is also evidence to suggest that the thalamus may also undergo structural changes, ${ }^{8}$ display functional abnormalities, ${ }^{9}$ and be a site of direct disease pathology in PD. ${ }^{1,10,11}$ Given that symptom presentation in PD only occurs after significant dopamine loss, it is important to examine the integrity of structures further into the direct basal ganglia pathway, such as the thalamus, particularly early in the disease.

While conventional MR imaging has limited utility as an investigative and diagnostic tool in $\mathrm{PD}$, recent advances in neuroimaging techniques have allowed for the noninvasive in vivo assessment of PD-related pathology. ${ }^{12,13}$ For example, DTI measures within the SN have shown excellent sensitivity and specificity in differentiating de novo patients with PD from healthy controls. ${ }^{14}$ Recent studies support the reduction in FA values within the $\mathrm{SN}$ of patients with $\mathrm{PD}^{15-17}$ and also suggest that mi- 
crostructural changes occur in areas beyond the $\mathrm{SN}$, including the thalamus. ${ }^{16}$ As a technique, DTI is based upon the diffusivity of water molecules, which is variably restricted by different tissues. FA values, which are calculated from the diffusion tensor images, range from 0 to 1 , with 0 representing isotropic diffusion and 1 anisotropic diffusion. In WM, where the tissue is highly organized, the free diffusion of water is limited, resulting in FA values near 1 . FA values calculated in WM reflect a combination of several factors, including axonal attenuation, degree of myelination, and orientational coherence. ${ }^{18,19}$ The direction of diffusion is less restricted in gray matter, resulting in FA values near 0 . It is unclear exactly what FA values represent in gray matter structures, but one hypothesis is that they reflect neuronal attenuation, with lower FA values representing a decrease in attenuation. ${ }^{20}$

Postmortem studies demonstrate that PD differentially affects specific thalamic nuclei, ${ }^{10,11,21-23}$ indicating that there could be degeneration or disruption of the microstructural integrity of the thalamus. To date, most studies utilizing DTI to investigate the thalamus have focused on the thalamus as a single entity and have not evaluated the integrity of specific nuclei or the critical corticalsubcortical fibers that project from the thalamus. ${ }^{15,16,24,25}$ Further, these studies examined relatively advanced patients with PD who had been taking antiparkinsonian medication, ${ }^{15,16,24,25}$ thus making it unclear whether differences between the patients and controls were caused by the disease, medication, or a combination of both.

In the current study, we conducted a detailed examination of the projection fibers from specific thalamic nuclei in de novo patients with $\mathrm{PD}$, allowing investigation of the disease without concern for the potential effects of antiparkinsonian medication. Using a high-resolution DTI sequence at 3T, we placed small ROIs in 6 thalamic regions and used the voxels within the ROIs as seeds for fiber tracking. Similar methods have been applied previously with high interrater reliability in a study of traumatic brain injury. ${ }^{26}$ Our hypothesis was that there would be degeneration of thalamic projection fibers in early stage, untreated PD, and that fibers from specific nuclei may be preferentially affected.

\section{MATERIALS AND METHODS \\ Subjects}

Forty individuals participated in this study: 20 patients with de novo PD (10 men, 10 women) and 20 age- and sex-matched healthy volunteers. There was no significant difference in age between the groups $(\mathrm{PD}=57.9 \pm 8.9$ years versus control $=58.2 \pm$ 9.6 years; $P=.7$ ). All patients were diagnosed with $\mathrm{PD}$ by a movement-disorders neurologist at Rush University Medical Center and met the United Kingdom PD Society Brain Bank diagnostic criteria. ${ }^{27,28}$ For the PD group, the mean (SD) Unified Parkinson Disease Rating Scale Part III motor score was 16.7 (7.6), indicating mild disease, and the mean (SD) disease duration in months was 12.8 (10.7). None of the controls reported a history of neuropsychiatric or neurologic problems. All subjects provided written informed consent for the procedures in this study, which were approved by the institutional review boards at Rush University Medical Center and the University of Illinois at Chicago.

\section{Image Acquisition}

To reliably perform FA analysis and fiber tracking in the thalamus, we used a high-resolution DTI protocol that was designed to minimize eddy-current-induced distortion. ${ }^{29}$ All images were collected on a 3T Signa HDx (GE Healthcare, Milwaukee, Wisconsin) using an 8-channel phased-array head coil, together with parallel imaging. The DTI data acquisition parameters were as follows: $\mathrm{TR}=4,500 \mathrm{~ms} ; \mathrm{TE}=82 \mathrm{~ms}$; flip angle $=90^{\circ}$; $\mathrm{b}$ values $=$ 0,1000 seconds $/ \mathrm{mm}^{2} ;$ diffusion directions $=27 ; \mathrm{FOV}=200$ $\mathrm{mm}^{2}$; image matrix size $=256 \times 256$; number of sections $=15$; section thickness $=4 \mathrm{~mm}$; section gap $=1 \mathrm{~mm} ; \mathrm{NEX}=4$; and acceleration factor $=2$. Thus, the voxel size was $0.78 \times 0.78 \times 4$ $\mathrm{mm}$. The images were collected in the axial orientation, with the top section placed approximately $4 \mathrm{~mm}$ above the corpus callosum. The total acquisition time of the DTI sequence was 8 minutes, 33 seconds.

To visualize the thalamus and differentiate it from surrounding structures, a set of T2-weighted images was acquired using a fast spin-echo pulse sequence in the axial plane (TR $=5000 \mathrm{~ms}$; $\mathrm{TE}=97 \mathrm{~ms}$; flip angle $=90^{\circ}$; FOV $=200 \mathrm{~mm}^{2}$; image matrix size $=512 \times 512 ;$ number of sections $=15$; section thickness $=4$ $\mathrm{mm}$; section gap $=1 \mathrm{~mm}$; NEX $=2$ ).

To help visualize the DM, we collected axial images using a T1-weighted 3D inversion recovery fast spoiled gradient recalled pulse sequence $\left(\mathrm{TR}=25 \mathrm{~ms}\right.$; $\mathrm{TE}=3 \mathrm{~ms}$; flip angle $=40^{\circ}$; FOV $=240 \mathrm{~mm}^{2}$; image matrix size $=256 \times 256$; number of sections $=120$; section thickness $=1.5 \mathrm{~mm}$; section gap $=0 \mathrm{~mm}$; $\operatorname{NEX}=1)$.

\section{Image Analysis}

DTIStudio (Johns Hopkins University, Baltimore, Maryland) ${ }^{30}$ was used to reconstruct the images and calculate FA values. For all subjects, each of the 15 diffusion tensor images was examined visually for artifacts related to eddy currents and motion, namely, spatial blurring, scaling, and shearing. Head motion was further assessed using $\mathrm{AFNI}^{31}$ software (http://afni.nimh.nih.gov/afni) and required to be within $1 \mathrm{~mm}$. A background noise level of 125 (MR units) was applied before calculation of the pixel-wise FA maps.

\section{Fiber Tracking}

Fiber tracking in DTIStudio ${ }^{32}$ was used to assess projection fibers from the 6 thalamic regions. After the calculation of pixel-wise FA and eigenvector maps, fiber tracking parameters were set to exclude tracking fibers with FA values $<0.20$ or a turning angle $>70^{\circ}$. For each subject, 2 circular ROIs ( $3 \mathrm{~mm}$ in diameter) were placed in the AN, VA, VL, VPL/VPM, and PU. Given its larger size, 3 ROIs of the same diameter were placed in the DM. ROIs were drawn independently by 2 raters who were blinded to patient or control status. Fig $1 A$ shows the regional boundaries for placing the ROIs, which were based on methods described by Little and colleagues (Appendix e-1). ${ }^{26}$ VPL and VPM were considered as one region due to the difficulty visualizing their boundaries. For each thalamic region and subject, mean FA values were calculated across the entire extent of the fibers identified by the ROI seed voxels. ROIs were drawn separately for the right and left hemispheres. 


\section{Placement of ROIs in Thalamic Regions}

Anterior Nucleus. The AN ROIs were defined laterally by the internal capsule, medially by the edge of the thalamus, and anteriorly by the posterior edge of the caudate head and lateral ventricle. The posterior boundary for the ROIs was defined by a horizontal line extending from the posterior edge of the anterior limb of the internal capsule. Any fiber that extended to the putamen or globus pallidus was excluded. Furthermore, any fibers that were also identified from ROIs placed in the VA, VPL/VPM, PU, or DM were excluded.

Ventral Anterior Nucleus. The anterior edges of the VA ROIs were defined by a horizontal line extending from the anterior edge of the internal capsule and extended medially the width of the internal capsule. The VA ROIs extended posterior to a horizontal line drawn from the posterior boundary of the putamen. Any fibers that were also identified by the AN ROIs were excluded.

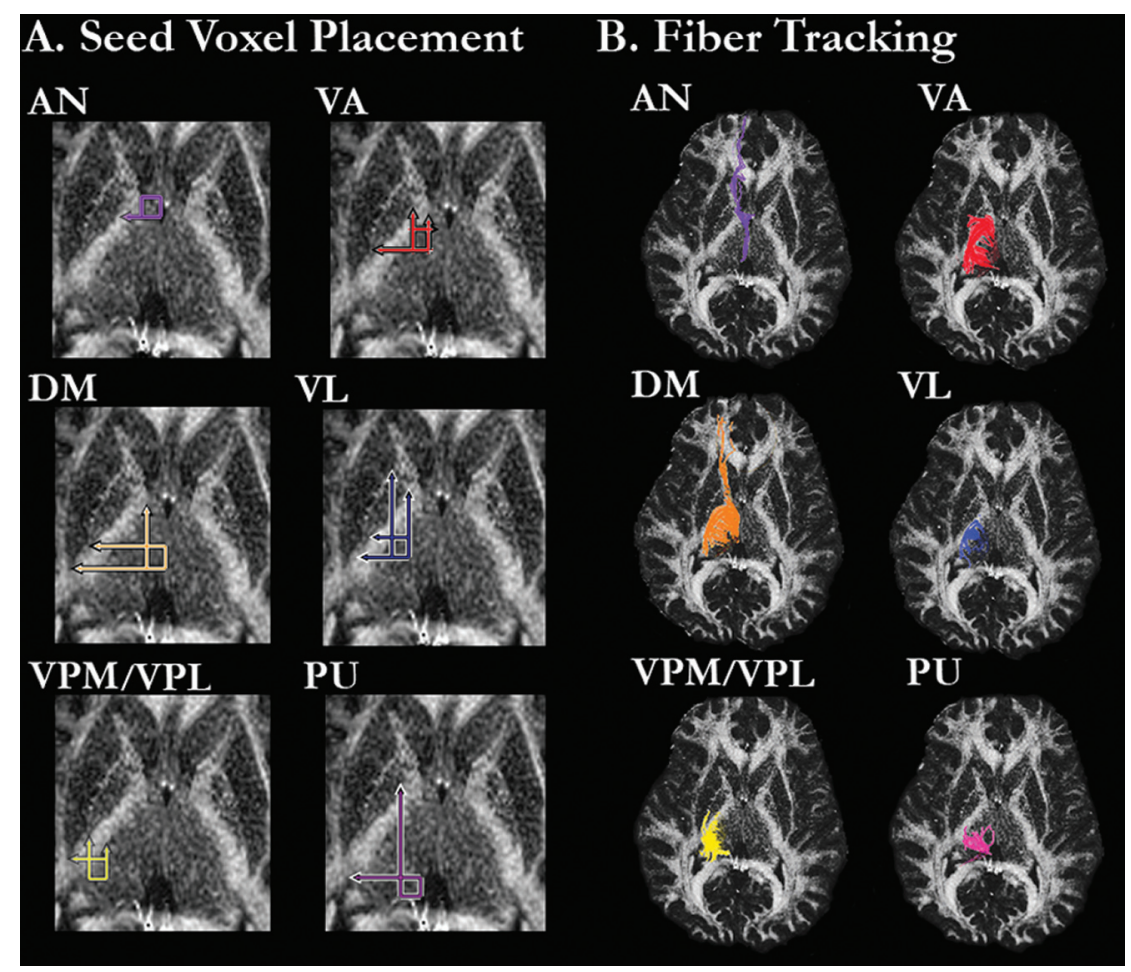

FIG 1. FA image from a representative healthy control subject showing $(A)$ regional boundaries of the 6 thalamic regions in which the circular ROls were placed, and (B) fiber tracking from the ROIs in each thalamic region.
Ventral Lateral Nucleus. The VL ROIs were defined medially by a vertical line drawn down from the medial edge of the bend of the internal capsule (the intersection between the anterior limb of the internal capsule and the body of the internal capsule), laterally by the edge of the internal capsule, anteriorly by a horizontal line extending from the posterior edge of the anterior limb of the internal capsule, and posteriorly by a horizontal line extending from the posterior boundary of the putamen. Fibers that were previously identified by either the AN or VA ROIs were excluded.

Dorsomedial Nucleus. The DM ROIs were defined medially by the edge of the thalamic body, laterally by a vertical line extending from the posterior edge of the anterior limb of the medial edge of the internal capsule, posteriorly by a horizontal line extending from the posterior edge of the posterior limb of the internal capsule, and anteriorly by a horizontal line extending from the posterior edge of the putamen. Fibers that were previously identified by the AN, VA, or VL ROIs were excluded.

Ventral Posterior Lateral and Ventral Posterior Medial Nuclei. The VPL/VPM ROI was defined anteriorly by a horizontal line extending from the posterior edge of the putamen, laterally by a vertical line extending from the posterior intersection of the internal capsule and putamen, medially by a vertical line extending the intersection of the anterior limb of the internal capsule with the anterior edge of the putamen, and posteriorly by the posterior edge of the thalamus. Fibers that were also identified by the AN, VA, VL, or DM ROIs were excluded.

Pulvinar. The PU ROIs were defined medially and posteriorly by the edge of the thalamus, laterally by a vertical line extending from the medial intersection of the anterior limb of the internal capsule and body of the internal capsule, and anteriorly by a horizontal line extending from the intersection of the posterior limb of the internal capsule and putamen. Fibers that were previously identified by the AN, VA, VL, VPL/VPM, or DM ROIs were excluded.

\section{Statistical Analysis}

For each thalamic region, FA values within each group were assessed for outliers. No FA value fell more than 3 SD from its respective mean; thus, all data were included in the analyses. Next, we performed a 2-way mixed design ANOVA on the FA values for each thalamic region, with group (PD, control) as the betweensubjects factor and hemisphere as the within-subjects factor (right, left). The assessment of interrater reliability for ROI placement showed strong agreement between the 2 raters, with all interclass correlation coefficients above 0.82 . Because the 2-way interaction did not approach significance for any analysis, we collapsed the data across the cerebral hemispheres. For thalamic regions that differed significantly in FA values between groups, we performed a correlation analysis between FA values and Unified Parkinson Disease Rating Scale Part III scores.

\section{RESULTS}

The 2-way interaction between group and hemisphere was not significant for any of the 6 thalamic regions (all $P_{\mathrm{s}}>.4$ ). The main effect of hemisphere was only significant for the VL $(P=.012)$, with higher FA values on the left than right side. There was a significant main effect of group for 3 of the 6 thalamic regions. As presented in Fig 2, FA values were reduced in the PD group compared with the control group for fibers projecting from the AN 


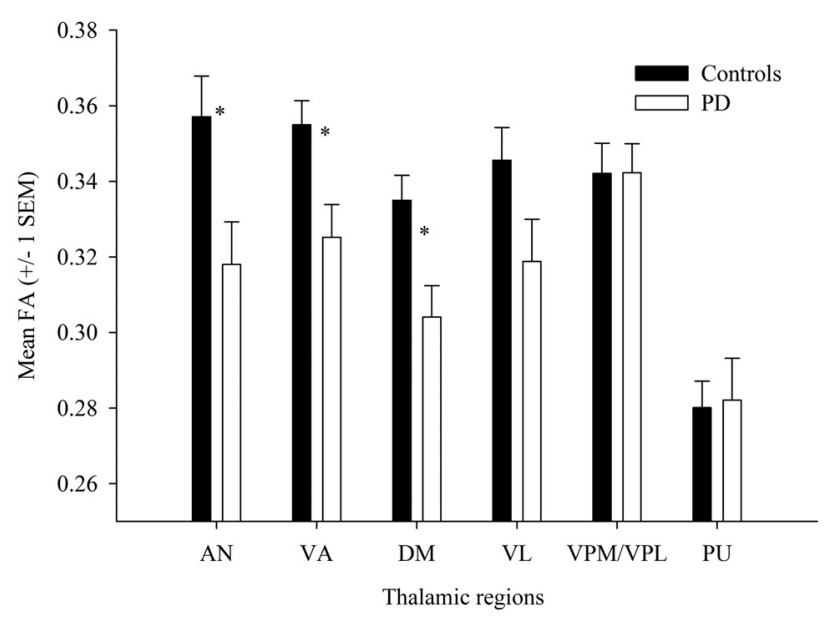

FIG 2. Mean ( \pm SEM) FA values from each of the 6 thalamic regions for the control and PD groups. Note that the PD group has significantly reduced of $F A$ values compared with the control group in the $\mathrm{AN}, \mathrm{VA}$, and DM, and shows a marginally significant reduction in the $\mathrm{VL}$.

$(P=.016)$, VA $(P=.009)$, and DM $(P=.006)$. In addition, the reduction in FA values from the VL approached significance forthe PD group compared with the control group $(P=.065)$. The FA values in the VPL/VPM and PU projection fibers did not differ significantly between the groups (both $P \mathrm{~s}>.4$ ).

For the 3 thalamic regions that differed significantly between the groups, we performed a correlation analysis to investigate the relationship between Unified Parkinson Disease Rating Scale Part III scores and FA values in PD. There was a significant correlation between Unified Parkinson Disease Rating Scale Part III scores and FA values in the AN $(r=-.487, P=.029)$, indicating a moderate negative association; as Unified Parkinson Disease Rating Scale Part III scores (ie, motor impairment) increased, FA values decreased. The correlations between Unified Parkinson Disease Rating Scale Part III scores and FA values in the DM, VA, and VL were not significant (all Ps $>.2$ ).

\section{DISCUSSION}

The thalamus plays an important role in the output pathway of the basal ganglia circuitry. It not only transmits nearly all cortical inputs, including cognitive, sensory, and motor information, but also modulates and integrates this information. Further, cerebellar output influences the cortical areas involved in motor processing via the thalamus. The thalamus is organized into distinct nuclear regions, from which diffuse and specific efferent projections are sent to cortical, cerebellar, and subcortical regions. ${ }^{3}$

The present study was the first to examine the integrity of thalamic nuclei projections in de novo PD using in vivo DTI tractography. We showed that there were reduced FA values in the fibers projecting from the AN, VA, and DM, but not the VPL/ VPM or PU, in patients with PD compared with controls. Further, the reduction in FA values approached significance in the VL of the patients with PD. Although the pathologic cause of these reduced FA values is not known, it may reflect demyelination, neuronal loss, or gliosis. ${ }^{20,32-35}$ We also showed that there was a significant negative correlation between FA values in the AN and Unified Parkinson Disease Rating Scale Part III scores. This rela- tionship should be investigated further in future work. Collectively, our results provide preliminary evidence of thalamic fiber degeneration in early stage PD and shed light on the extent of disrupted thalamic circuitry as a result of the disease.

Our DTI results are largely consistent with postmortem studies of the thalamus in PD. Rüb et $\mathrm{al}^{11}$ reported evidence of mild inclusion pathology in the AN, VA, VL, and DM of patients with mild to severe PD. Further, there was no evidence of pathology in the VPM and VPL, and relatively little pathology in the PU of these patients. However, other studies have reported that the volume and number of neurons in the AN and DM are not reduced significantly in PD. ${ }^{21-23}$

The AN, VA, and DM are considered specific nuclei, in that they relay information to functionally distinct cortical regions. The AN is an associative nucleus that is part of the major limbic pathway, and primarily projects information related to emotion and memory to the cingulate gyrus. ${ }^{36}$ Similarly, the DM is involved in emotion ${ }^{37}$ and cognition, and is connected to the amygdala $^{38}$ and the anterior cingulate and prefrontal cortices. ${ }^{36,39,40}$ The VA, which, in combination with the VL, is known as the "motor thalamus," primarily relays somatomotor information to the supplementary motor area from the basal ganglia. In functional imaging studies of PD, the supplementary motor area is often hypoactive during movement tasks compared with controls. ${ }^{41,42}$ Taken together, damage to these different thalamic projection fibers may result in widespread impairments ${ }^{38,43}$ and may contribute to symptom presentation in PD.

Although ex vivo studies remain the criterion standard for pathologic confirmation of $\mathrm{PD}$, it is important to study the brain in vivo to gain insight into the structures affected early in the disease and to evaluate differences across disease stages. As a technique, DTI has proved useful in identifying structural abnormalities in the $\mathrm{SN}$ of patients with PD in vivo. ${ }^{14,15,17,24,44}$ However, DTI studies comparing the thalamus between patients with PD and controls have been less conclusive. In a large cohort, Chan et $\mathrm{al}^{24}$ did not observe differences in FA values at 1.5T between mild to severe patients with PD on antiparkinsonian medication and controls in a ROI that covered a single section of the thalamus. Similarly, there were no differences in mean diffusivity values (FA values were not reported) at $1.5 \mathrm{~T}$ in a circular ROI placed on 1 section of the thalamus between early-stage medicated patients with PD and controls. ${ }^{45}$ However, in another large cohort, Péran et $\mathrm{al}^{15}$ reported reduced FA and mean diffusivity values at $3 \mathrm{~T}$ in the whole thalamus of early stage, but medicated, patients with PD and controls. Most recently, a DTI study that examined the thalamus using both voxelwise and ROI-based approaches reported reduced FA values at $4 \mathrm{~T}$ in mild to severe medicated patients with PD versus controls. ${ }^{16}$ Collectively, these studies suggest that magnet field strength and/or ROI size and placement may affect our ability to detect microstructural changes in the thalamus.

As discussed earlier, the nuclei of the thalamus have specialized connectivity and functions, and thus may be differentially affected in disease states, as supported by postmortem research. By studying the thalamus as a single entity, it is unclear whether significant effects in large ROIs or the whole thalamus are driven by degeneration in a few select nuclei, and whether nonsignificant effects are the result of placing small ROIs on relatively spared nuclei. The present study addressed these concerns using high-resolution fiber tracking at 3T, allowing for specific thalamic nuclei to be studied with great sensitiv- 
ity. Indeed, we showed that FA values were reduced in the AN, VA, and DM in the patients with PD compared with controls. There was also a marginally significant reduction in FA values from the VL projections of the patients with PD.

Although DTI has proven useful in studying subregions of the SN in $\mathrm{PD},{ }^{14}$ relatively few studies have used this technique to examine individual thalamic nuclei. In fact, to date, only the DM and VL have been examined in PD. Consistent with the role of the DM in affect and cognition, $\mathrm{Li}$ et $\mathrm{al}^{25}$ reported that patients with $\mathrm{PD}$ with depression had reduced FA values compared with nondepressed patients with $\mathrm{PD}$, and that these FA values correlated negatively with depression severity. Given that depression is prevalent in $\mathrm{PD},{ }^{46}$ it is possible that the patients in our study had greater affective disturbances than the controls. The VL segment of the motor thalamus receives input from the cerebellum ${ }^{47}$ and basal ganglia and projects primarily to the pre- and primary motor cortices, retaining its topographic organization throughout. Two previous MR imaging studies at $1.5 \mathrm{~T}$ have examined the VL in PD, neither of which showed differences in DTI measures between the PD and control groups. ${ }^{44,48}$ In the present study, the difference in FA values between the 2 groups approached significance in the VL. Using a high field strength scanner to test larger samples may increase the ability to detect microstructural changes in the VL.

There were 2 thalamic regions with FA values that did not differ significantly between the groups, namely, the VPL/VPM and PU, both of which are sensory nuclei. Human DTI tractography has shown that the PU is connected ipsilaterally to several cortical and subcortical areas, particularly those in the visual pathways. ${ }^{49}$ Although its function is not completely understood, the PU is thought to play an important role in higher order visual processing and integration, including visual attention and oculomotor behavior. ${ }^{50}$ The VPL and VPM relay touch and proprioceptive information from the contralateral body and head, respectively, to the primary and secondary somatosensory cortices. ${ }^{40}$ The fact that we did not show a significant group difference in the PU or VPL/VPM suggests that visual and somatosensory processing should be relatively intact in early-stage PD.

There are a few limitations to the present study. First, a detailed neuropsychologic examination was not performed on the patients with PD. Given that cognitive and affective problems occur frequently in $\mathrm{PD},{ }^{47}$ and that we found differences in thalamic nuclei known to be involved in emotion and cognitive processing, it is important to collect these data in the future to better understand the relationship between thalamic degeneration and these nonmotor functions in PD. Second, the reduction of FA values in the VL of patients with PD did not quite reach significance, suggesting that larger sample sizes or more impaired patients may be necessary to detect a difference in this nucleus. Third, intralaminar and midline nuclei (eg, centromedian-parafasicular complex) were not examined because their boundaries could not be distinguished on the MR imaging scans with acceptable interrater reliability. These nuclei, which are involved in arousal and awareness, ${ }^{51}$ have shown severe pathology in postmortem studies of PD. ${ }^{11,21-23}$ Fourth, data in superior cortical areas were not collected due to limitations in the number of sections that can be collected within a reasonable acquisition time. This limited quantification of the tract projections.

\section{CONCLUSIONS}

The present study provides the first in vivo evidence that there is a disruption of the projection fibers in certain thalamic nuclei, namely the AN, VA, and DM, in de novo patients with PD. These results suggest that structural degeneration occurs beyond the $\mathrm{SN}$ in early PD and thus help further our understanding of the neural structures affected by the disease. Specifically, we showed that nuclei involved in motor, cognitive, and affective processes are disrupted, whereas those involved in sensory processes are relatively spared. In addition, this study showed that the current methodology has utility in examining the microstructural integrity of thalamic nuclei in not only traumatic brain injury ${ }^{26}$ but also PD. Future research should use these methods longitudinally to determine whether thalamic fiber integrity changes with disease progression and whether it is affected by antiparkinsonian medication. Further, a detailed neuropsychologic examination should be conducted along with DTI of thalamic regions to better understand the impact of fiber degeneration on cognitive, affective, and behavioral functioning in PD.

\section{ACKNOWLEDGMENTS}

We thank the staff at the Center for MR Research at UIC for their assistance with data collection, the staff at the Movement Disorder Center at Rush University Medical Center for their help with patient recruitment, and all of the participants for their time and commitment to this research. The contents of this manuscript are solely the responsibility of the authors and do not necessarily represent the official views of the National Institute of Neurologic Disorders and Stroke, Department of Veterans Affairs, United States Government, UF, UIC, Scott \& White Healthcare, or Texas A\&M Health Sciences.

Disclosures: Daniel Corcos—RELATED: Grant: NIH;* Support for Travel to Meetings for the Study or Other Purposes: NIH.* Deborah Little_-RELATED: Grant: Department of Defense, ${ }^{*}$ Department of Veterans Affairs. ${ }^{*}$ David Vaillancourt-RELATED: Grant: Scaling and Sequencing Motor Output in Humans, ${ }^{*}$ Comments: NIH RO1 (I am PI); UNRELATED: Consultancy: I consult on a project at Northwestern University using diffusion imaging in Parkinson's disease. The project is unrelated to the current project; Grants/Grants Pending: NIH R01 N5058487-05, Comments: Role of Cortex and Cerebellum in Visually-Guided Motor Behavior.* (*Money paid to institution)

\section{REFERENCES}

1. Braak H, Del Tredici K, Rüb U, et al. Staging of brain pathology related to sporadic Parkinson's disease. Neurobiol Aging 2003;24: 197-211

2. Hodaie M, Neimat JS, Lozano AM. The dopaminergic nigrostriatal system and Parkinson's disease: molecular events in development, disease, and cell death, and new therapeutic strategies. Neurosurgery 2007;60:17-28

3. Blumenfeld H. Neuroanatomy through Clinical Cases. Sunderland, Massachusetts: Sinauer Associates; 2002

4. Owen AM, Doyon J, Dagher A, et al. Abnormal basal ganglia outflow in Parkinson's disease identified with PET. Implications for higher cortical functions. Brain 1998;121:949-65

5. Braak H, Braak E. Pathoanatomy of Parkinson's disease. J Neurol 2000;247:3-10

6. Emre M. What causes mental dysfunction in Parkinson's disease? Mov Disord 2003;18:S63-71

7. Nobili F, Campus C, Arnaldi D, et al. Cognitive-nigrostriatal relationships in de novo, drug-naïve Parkinson's disease patients: a [I123]FP-CIT SPECT Study. Mov Disord 2010;25:35-43

8. McKeown MJ, Uthama A, Abugharbieh R, et al. Shape (but not vol- 
ume) changes in the thalami in Parkinson disease. BMC Neurol 2008;8:8

9. Prodoehl J, Spraker M, Corcos D, et al. Blood oxygenation leveldependent activation in basal ganglia nuclei relates to specific symptoms in de novo Parkinson's disease. Mov Disord 2010;25: 2035-43

10. Halliday GM. Thalamic changes in Parkinson's disease. Parkinsonism \& Relat Disord 2009;15:S152

11. Rüb U, Del Tredici K, Schultz C, et al. Parkinson's disease: the thalamic components of the limbic loop are severely impaired by asynuclein immunopositive inclusion body pathology. Neurobiol Aging 2002;23:245-54

12. Planetta PJ, Prodoehl J, Corcos DM, et al. Use of MRI to monitor Parkinson's disease. Neurodegener Dis Manag 2011;1:67-77

13. Mahlknecht P, Hotter A, Hussl A, et al. Significance of MRI in diagnosis and differential diagnosis of Parkinson's disease. Neurodegener Dis 2010;7:300-18

14. Vaillancourt D, Spraker M, Prodoehl J, et al. High-resolution diffusion tensor imaging in the substantia nigra of de novo Parkinson disease. Neurology 2009;72:1378-84

15. Péran P, Cherubini A, Assogna F, et al. Magnetic resonance imaging markers of Parkinson's disease nigrostriatal signature. Brain 2010; 133:3423-33

16. Zhan W, Kang GA, Glass GA, et al. Regional alterations of brain microstructure in Parkinson's disease using diffusion tensor imaging. Mov Disord 2012;27:90-97

17. Du G, Lewis MM, Styner M, et al. Combined r2* and diffusion tensor imaging changes in the substantia nigra in Parkinson's disease. Mov Disord 2011;26:1627-32

18. Basser PJ, Mattiello J, Lebihan D. MR diffusion tensor spectroscopy and imaging. Biophys J 1994;66:259-67

19. Minati L, Banasik T, Brzezinski J, et al. Elevating tensor rank increases anisotropy in brain areas associated with intra-voxel orientational heterogeneity (IVOH): a generalised DTI (GDTI) study. NMR Biomed 2008;21:2-14

20. Boska MD, Hasan KM, Kibuule D, et al. Quantitative diffusion tensor imaging detects dopaminergic neuronal degeneration in a murine model of Parkinson's disease. Neurobiol Dis 2007;26:590-96

21. Brooks D, Halliday GM. Intralaminar nuclei of the thalamus in Lewy body diseases. Brain Res Bull 2009;78:97-104

22. Henderson JM, Carpenter K, Cartwright $\mathrm{H}$, et al. Degeneration of the centre median-parafascicular complex in Parkinson's disease. Ann Neurol 2000;47:345-52

23. Henderson JM, Carpenter K, Cartwright H, et al. Loss of thalamic intralaminar nuclei in progressive supranuclear palsy and Parkinson's disease: clinical and therapeutic implications. Brain 2000;123: $1410-21$

24. Chan LL, Rumpel H, Yap K, et al. Case control study of diffusion tensor imaging in Parkinson's disease. J Neurol Neurosurg Psychiatry 2007;78:1383-86

25. Li W, Liu J, Skidmore F, et al. White matter microstructure changes in the thalamus in Parkinson disease with depression: a diffusion tensor MR imaging study. AJNR Am J Neuroradiol 2010;31:1861-66

26. Little DM, Kraus MF, Joseph J, et al. Thalamic integrity underlies executive dysfunction in traumatic brain injury. Neurology 2010;74: $558-64$

27. Hughes A, Daniel S, Kilford L, et al. Accuracy of clinical diagnosis of idiopathic Parkinson's disease: a clinico-pathological study of $\mathbf{1 0 0}$ cases. J Neurol Neurosurg Psychiatry 1992;55:181-84

28. Hughes AJ, Ben-Shlomo Y, Daniel SE, et al. What features improve the accuracy of clinical diagnosis in Parkinson's disease: a clinicopathologic study. 1992. Neurology 2001;57:S34-38

29. Zhou X, Naier J, Reynolds H. Method to reduce eddy current effects in diffusion-weighted echo planar imaging. United States Patent, 5 1999;864:233

30. Jiang H, van Zijl PC, Kim J, et al. DTIStudio: resource program for diffusion tensor computation and fiber bundle tracking. Comput Methods Programs Biomed 2006;81:106-16
31. Cox RW. AFNI: software for analysis and visualization of functional magnetic resonance neuroimages. Comput Biomed Res 1996; 29:162-73

32. DeBoy CA, Zhang J, Dike S, et al. High resolution diffusion tensor imaging of axonal damage in focal inflammatory and demyelinating lesions in rat spinal cord. Brain 2007;130:2199-210

33. Budde MD, Janes L, Gold E, et al. The contribution of gliosis to diffusion tensor anisotropy and tractography following traumatic brain injury: validation in the rat using Fourier analysis of stained tissue sections. Brain 2011;134:2248-60

34. Wimberger DM, Roberts TP, Barkovich AJ, et al. Identification of "premyelination" by diffusion-weighted MRI. J Comput Assist Tomogr 1995;19:28-33

35. Klingberg T, Vaidya CJ, Gabrieli JD, et al. Myelination and organization of the frontal white matter in children: a diffusion tensor MRI study. Neuroreport 1999;10:2817-21

36. Zhang D, Snyder AZ, Shimony JS, et al. Noninvasive functional and structural connectivity mapping of the human thalamocortical system. Cereb Cortex 2010;20:1187-94

37. Metzger CD, Eckert U, Steiner J, et al. High field FMRI reveals thalamocortical integration of segregated cognitive and emotional processing in mediodorsal and intralaminar thalamic nuclei. Front Neuroanat 2010;4:138

38. Schmahmann JD. Vascular syndromes of the thalamus. Stroke 2003; 34:2264-78

39. Klein JC, Rushworth MF, Behrens TE, et al. Topography of connections between human prefrontal cortex and mediodorsal thalamus studied with diffusion tractography. Neuroimage 2010;51:555-64

40. Behrens TE, Johansen-Berg H, Woolrich MW, et al. Non-invasive mapping of connections between human thalamus and cortex using diffusion imaging. Nat Neurosci 2003;6:750-57

41. Jahanshahi M, Jenkins IH, Brown RG, et al. Self-initiated versus externally triggered movements. I. An investigation using measurement of regional cerebral blood flow with PET and movement-related potentials in normal and Parkinson's disease subjects. Brain 1995;118:913-33

42. Spraker M, Prodoehl J, Corcos D, et al. Basal ganglia hypoactivity during grip force in drug naïve Parkinson's disease. Hum Brain Mapp 2010;31:1928-41

43. Carrera E, Bogousslavsky J. The thalamus and behavior: effects of anatomically distinct strokes. Neurology 2006;66:1817-23

44. Yoshikawa K, Nakata Y, Yamada K, et al. Early pathological changes in the parkinsonian brain demonstrated by diffusion tensor MRI. J Neurol Neurosurg Psychiatry 2004;75:481-84

45. Gattellaro G, Minati L, Grisoli M, et al. White matter involvement in idiopathic Parkinson disease: a diffusion tensor imaging study. AJNR Am J Neuroradiol 2009;30:1222-26

46. Chaudhuri KR, Healy DG, Schapira AH, et al. Non-motor symptoms of Parkinson's disease: diagnosis and management. Lancet Neurol 2006:5:235-45

47. Kwon HG, Hong JH, Hong CP, et al. Dentatorubrothalamic tract in human brain: diffusion tensor tractography study. Neuroradiology 2011;53:787-91

48. Nicoletti G, Manners D, Novellino F, et al. Diffusion tensor MRI changes in cerebellar structures of patients with familial essential tremor. Neurology 2010;74:988-94

49. Leh SE, Chakravarty MM, Ptito A. The connectivity of the human pulvinar: a diffusion tensor imaging tractography study. Int J Biomed Imaging 2008:78953

50. Arend I, Machado L, Ward R, et al. The role of the human pulvinar in visual attention and action: evidence from temporal-order judgment, saccade decision, and antisaccade tasks. Prog Brain Res 2008; 171:475-83

51. Van der Werf YD, Witter MP, Groenewegen HJ. The intralaminar and midline nuclei of the thalamus. Anatomical and functional evidence for participation in processes of arousal and awareness. Brain Res Brain Res Rev 2002;39:107-40 